\title{
Radiogenomics of medulloblastoma: imaging surrogates of molecular biology
}

\author{
Archya Dasgupta, Tejpal Gupta \\ Department of Radiation Oncology, ACTREC, Tata Memorial Centre, Navi Mumbai 410210, India.
}

Correspondence to: Dr. Tejpal Gupta, Department of Radiation Oncology, ACTREC, Tata Memorial Centre, Navi Mumbai 410210, India. E-mail: tejpalgupta@rediffmail.com

How to cite this article: Dasgupta A, Gupta T. Radiogenomics of medulloblastoma: imaging surrogates of molecular biology. J Trans/ Genet Genom 2018;2:15. https://doi.org/10.20517/jtgg.2018.21

Received: 7 Jul 2018 First Decision: 17 Jul 2018 Revised: 13 Sep 2018 Accepted: 17 Sep 2018 Published: 24 Oct 2018

Science Editor: David N. Cooper Copy Editor: Cui Yu Production Editor: Zhong-Yu Guo

\begin{abstract}
Medulloblastoma is a heterogeneous disease comprising four molecular subgroups - wingless (WNT), sonic hedge hog $(\mathrm{SHH})$, group 3, and group 4, with distinct developmental origins, unique transcriptional profiles, diverse phenotypes, and varying clinical outcomes. Magnetic resonance imaging (MRI) is the preferred first-line imaging modality in the diagnosis and staging of suspected brain tumors including medulloblastoma. It is being increasingly recognized that imaging features reflect underlying disease biology that can serve as independent predictive and prognostic biomarkers. Radiogenomics is an emerging field of research that aims to define relationships between non-invasive imaging features (radio-phenotypes) and genomic data/molecular markers (molecular phenotypes). Recent studies have reported encouraging data regarding imaging genomics of medulloblastoma with certain MRI features correlating with specific molecular subgroups. These include lateralized cerebellar location for $\mathrm{SHH}$-subgroup; cerebellopontine angle location for WNT-subgroup; and inferior location with dilation of superior recess of the IVth ventricle for group 4 tumors. Minimal enhancement of primary tumor and ependymal metastases (infundibular/suprasellar) with mismatching pattern is a specific feature of group 4 medulloblastoma. A 5-metabolite signature profile on magnetic resonance spectroscopy reliably differentiates $\mathrm{SHH}$-subgroup from non-WNT/non-SHH medulloblastoma. SHH-specific binary nomogram (location on horizontal and vertical axis, relationship with dorsal brainstem, pattern of contrast-enhancement, and peri-tumoral edema as discriminating imaging features) is associated with excellent predictive accuracy, followed by group 4-specific nomogram, with suboptimal accuracy of WNT and group 3-specific nomograms. The advent of deep machine-learning techniques and convoluted artificial neural networks should provide unique opportunities to further improve the accuracy of such radiogenomic correlation and prediction.
\end{abstract}

Keywords: Genomics, imaging, medulloblastoma, molecular subgrouping 


\section{INTRODUCTION}

Medulloblastoma, the most common pediatric malignant neoplasm of the central nervous system (CNS) comprising $20 \%-25 \%$ of all childhood brain tumors ${ }^{[1,2]}$ belongs to the primitive embryonal group of tumors that arises in the posterior fossa and is classified histologically as World Health Organization (WHO) grade IV tumor. Over the last decade or so, novel biological insights into its heterogeneity have led to the identification of various molecular subgroups with distinct developmental origins, unique transcriptional profiles, diverse phenotypes, and variable clinical outcomes ${ }^{[3-6]}$. The consensus classification ${ }^{[6]}$ comprising four distinct molecular subgroups - wingless (WNT), sonic hedge hog (SHH), Group 3, and Group 4 medulloblastoma has now been incorporated in the 2016 update of the WHO classification of CNS tumors $^{[7]}$. With rapid evolution of genomic technology, several platforms/methodologies are now available for molecular subgrouping of medulloblastoma. These include expression profiling of a select set of marker genes at RNA level using nanoString ${ }^{[8]}$ or real time reverse transcriptase polymerase chain reaction ${ }^{[9,10]}$; differential expression of select set of protein-coding genes and microRNAs $s^{[11]}$; expression of selected marker proteins ${ }^{[12,13]}$ on immunohistochemistry (IHC); and DNA methylation array ${ }^{[14,15]}$. Each of the methodologies have their unique advantages and disadvantages with its selection left to the judgement and discretion of the treating physician based on available resources (infrastructure and expertise), cost, and turn-around time.

\section{Conventional imaging features of medulloblastoma}

In patients with suspected brain tumors, a computed tomography (CT) scan and/or magnetic resonance imaging (MRI) of the brain is required to arrive at a presumptive diagnosis and guide further decisionmaking. Given the high propensity of neuraxial spread in medulloblastoma via cerebrospinal fluid pathways, pre-operative imaging of the spine is also recommended in the initial diagnostic work-up of children with posterior fossa tumors ${ }^{[16,17]}$. The features of medulloblastoma on conventional neuro-imaging have been very well characterized and described in the indexed medical literature ${ }^{[16,17]}$. Medulloblastoma is generally seen as a well-defined, solid, hyper-dense lesion on plain CT that shows variable enhancement post-contrast, it arises typically from the vermis in the midline posterior fossa and fills the fourth ventricle causing obstructive hydrocephalus; less commonly it is located laterally in the cerebellar hemisphere with or without extension to the foramen. MRI with its exquisite anatomic resolution, multi-parametric nature, and ability to image the entire neuraxis in one session is the preferred imaging modality for suspected medulloblastoma. On T1weighted images, medulloblastoma generally appears iso- to hypo-intense compared to surrounding white matter. It exhibits variable signal intensity on T2-weighted images with densely cellular component of the tumor being hypo-intense and lesser cellular areas being iso-intense compared to surrounding white matter. Intra-tumoral or peri-tumoral cysts, if any, appear hyper-intense while calcification generally exhibits low signal on T2-weighted sequences. Following intravenous gadolinium, medulloblastoma shows significant but variable and heterogeneous contrast enhancement. Due to densely packed cells, medulloblastoma causes restriction of diffusion with correspondingly low apparent diffusion co-efficient values.

\section{Radiogenomics or imaging genomics}

Traditionally, imaging has been used for diagnosis (characterization of lesion) and staging (assessment of disease extent) in oncology. With regards to brain tumors, the neuro-radiology community has predominantly focussed on correlating imaging features with histomorphology and grading. However, as is now widely believed, images are more than just pictures ${ }^{[1,19]}$ that reflect underlying disease biology including the dynamics of complex biological processes such as gene expression, proliferation, metabolism, and angiogenesis. Radiogenomics or imaging genomics ${ }^{[20]}$ is an exciting and emerging field of research that aims to define relationships between non-invasive imaging features (radio-phenotypes) and genomic data/ molecular markers (molecular phenotypes). In the past few years, it has become increasingly possible to extract meaningful information from routine images that can be utilized beyond the diagnostic and staging roles of radiology. Radiogenomics is a multi-step process comprising sequential stages of image acquisition, image segmentation, feature selection, feature extraction, qualification and final correlation with molecular 
markers ${ }^{[18-20]}$. In its simplest form, semantic imaging features like size/volume, margins/borders, density or intensity characteristics, contrast enhancement, edema, necrosis, hemorrhage, calcification, can be assessed and quantified visually for correlation with molecular markers either singly or in combination. Introduction of computer-based algorithms has not only refined the assessment of semantic features, but also allows extraction of agnostic features (histograms, textures, wavelets, and fractal dimensions), which generally go beyond human capability. Such automated high-throughput processing significantly reduces time and virtually eliminates inter-observer variability often associated with human interpretation. This review dissects and discusses the current state of knowledge regarding the radiogenomics of medulloblastoma.

\section{Specific imaging features in medulloblastoma subgroups}

In the last decade or so, several investigators from across the world have tried to correlate semantic imaging features with molecular subgrouping in medulloblastoma. All such studies ${ }^{[21-31]}$ reporting on the imaging genomics of medulloblastoma are briefly summarized in Table 1. While it is quite clear that no single imaging feature is pathognomonic of any particular subgroup, certain imaging characteristics are much more prevalent in one subgroup compared to others and may even be highly specific for an individual molecular subgroup. Characteristic imaging features typically seen in each of the four individual molecular subgroups are summarized in Table 2 and illustrated in Figures 1-4.

\section{WNT SUBGROUP MEDULLOBLASTOMA}

Reportedly the rarest subgroup (comprising approximately $10 \%$ of all medulloblastoma), the WNTpathway medulloblastomas have the best prognosis amongst all four subgroups with 5-year overall survival exceeding $90 \%$ in almost all studies ${ }^{[3-6]}$. They are mostly uniform in their genetic aberrations, histological pattern, and clinical presentation. Classic histology is seen in the vast majority of WNTpathway medulloblastoma (> 95\%), although occasional large-cell/anaplastic (LCA) variants have also been reported $^{[5,6]}$. They are equally distributed amongst boys and girls and commonly seen in older children and teenagers, but rarely ever in infants ${ }^{[3,6]}$.

\section{Anatomic location}

It is hypothesized that the WNT-pathway medulloblastomas arise from neural progenitors in the dorsal brainstem nuclei ${ }^{[32,33]}$ which explains its midline location and close proximity to the dorsal brainstem. In one of the first clinical observations of an association between molecular subgrouping and tumor location, Teo et al. ${ }^{[21]}$, reported $100 \%$ of WNT-subgroup medulloblastomas $(n=5)$ as having a midline vermian location. Subsequently, Perreault and colleagues ${ }^{[22]}$ reported $75 \%$ of WNT-subgroup tumors $(n=14)$ as being situated along the cerebellar peduncle and even extending up to the cerebellopontine angle (CPA) cistern in accordance with their origin from brainstem nucleus. In another study, Wefers et al ${ }^{[23]}$ reported $75 \%$ of WNT-subgroup medulloblastomas $(n=8)$ touching the cuneate nucleus located in the medulla. Interestingly, they also found these tumors to be more frequently caudal as opposed to rostral, concordant with the mouse model study ${ }^{[32]}$, wherein the genes associated with WNT-pathway are more expressed in the lower rhombic lip as compared to the upper rhombic lip. The midline location (83\%) with extension into CPA (17\%) was also reported by Lastowska and colleagues ${ }^{[24]}$ in WNT-pathway medulloblastoma. In an imaging study exclusively involving children with WNT-subgroup medulloblastoma $(n=16)$, Patay et al. ${ }^{[26]}$ reported the involvement of specific sites such as foramen of Luschka (75\%), IVth ventricle (69\%), cisterna magna (31\%) and CPA cistern (19\%). The anatomic location of WNT-subgroup tumors was found to be similar even in adult WNT-subgroup tumors $(n=4)$, with $75 \%$ being or reaching the midline in the IVth ventricle ${ }^{[27]}$. In an exclusive cohort of adult medulloblastomas, Zhao et al. ${ }^{[28]}$ reported midline vermian location in 5 of 17 (29\%) WNT-pathway tumors and lateral extension into cerebellar hemisphere or peduncle in the remaining 12 (71\%) patients. All 17 adults WNT-pathway medulloblastomas had brainstem contact with cochlear or cuneate nucleus or both. In a cohort of 15 WNT-subgroup medulloblastoma, Mata-Mbemba et al ${ }^{[29]}$, reported the tumor to be confined in midline vermian location in $33 \%$ of children, with the remaining $67 \%$ showing extension either unilaterally or bilaterally along the lateral recess of the IVth ventricle and beyond reaching 
Table 1. Brief summary of studies correlating imaging features with molecular subgrouping in medulloblastoma

\begin{tabular}{|c|c|c|c|c|}
\hline Author \& year & Population & $\begin{array}{c}\text { Number of } \\
\text { patients }\end{array}$ & Imaging features & Key findings from the study \\
\hline Teo et al. ${ }^{[21]}, 2013$ & Pediatric MB & 60 & $\begin{array}{l}\text { Conventional MRI } \\
\text { (location) }\end{array}$ & $\begin{array}{l}\text { Hemispheric location was associated with } \mathrm{SHH}-\mathrm{MB}(>50 \%) \\
\text { Non-WNT/non-SHH tumors were lateralised in only } 5 \%\end{array}$ \\
\hline $\begin{array}{l}\text { Perreault et al. }{ }^{[22]}, \\
2014\end{array}$ & $\begin{array}{l}\text { Pediatric and } \\
\text { adult } \mathrm{MB}\end{array}$ & 99 & $\begin{array}{l}\text { Conventional MRI } \\
\text { features; diffusion- } \\
\text { weighted imaging } \\
\text { including apparent } \\
\text { diffusion coefficient } \\
\text { (ADC maps) }\end{array}$ & $\begin{array}{l}\text { Group } 3 \& 4 \text { tumors were predominantly located in the midline within the } \\
\text { IVth ventricle } \\
\text { WNT tumors were localised to CP/CPA cistern } \\
\text { SHH tumors were having cerebellar hemispheric location } \\
\text { Midline group } 4 \text { tumors had no or minimal enhancement } \\
\text { MRI based regression model correctly predicted subgroup in } 65 \% \\
\text { Mean ADC was not significantly different among the subgroups }\end{array}$ \\
\hline $\begin{array}{l}\text { Wefers et al. }{ }^{[23]}, \\
2014\end{array}$ & $\begin{array}{l}\text { Pediatric and } \\
\text { adult } \mathrm{MB}\end{array}$ & 71 & $\begin{array}{l}\text { Conventional MRI } \\
\text { (location and } \\
\text { relationship of tumor } \\
\text { with surrounding } \\
\text { structures) }\end{array}$ & $\begin{array}{l}\text { Subgroup with exclusive intracerebellar growth was } \mathrm{SHH}(52 \%) \\
\text { Most group } 3 \& 4 \text { tumors grew in the midline in vermis (>70\%) } \\
\text { A third of non-SHH tumors had tumor in caudal cerebellum } \\
\text { Brainstem contact was seen in only } 48 \% \text { of } \mathrm{SHH}-\mathrm{MB} \text { as compared to } 75 \% \\
\text { or more for other subgroups }\end{array}$ \\
\hline $\begin{array}{l}\text { Lastowska et al. }{ }^{[24]}, \\
2015\end{array}$ & Pediatric MB & 76 & $\begin{array}{l}\text { MRI (location } \\
\text { and contrast } \\
\text { enhancement } \\
\text { features) }\end{array}$ & $\begin{array}{l}\text { Lateralized location was significantly associated with } \mathrm{SHH} \\
\text { None of } \mathrm{SHH} \text { tumors were invading the brainstem floor } \\
\text { All group } 3 \text { tumors were associated with extensive contrast enhancement } \\
\text { (> } 75 \% \text { of tumor showing contrast uptake) } \\
\text { Over } 60 \% \text { of group } 4 \text { tumors were having no or minimal contrast } \\
\text { enhancement (< } 10 \% \text { of tumor showing contrast uptake) } \\
\text { For non-WNT/non-SHH tumors, extensive contrast uptake was associated } \\
\text { with poor survival }\end{array}$ \\
\hline $\begin{array}{l}\text { Bluml et al. }{ }^{[25]}, \\
\end{array}$ & Pediatric MB & 30 & MRS & $\begin{array}{l}\text { SHH-MB was associated with prominent choline/lipid peaks, low } \\
\text { creatinine, and little or no taurine levels. } \\
\text { Group } 3 \& 4 \text { tumors were characterized by low lipd levels, high creatinine, } \\
\text { and readily detected taurine } \\
\text { The 5-metabolite MRS signature reliably differentiated SHH-MB from } \\
\text { group 3/group } 4 \text { tumors }\end{array}$ \\
\hline $\begin{array}{l}\text { Patay et al. }{ }^{[26]}, \\
2015\end{array}$ & WNT MB & 16 & $\begin{array}{l}\text { Conventional MRI } \\
\text { features (also } \\
\text { assessed post-surgery } \\
\text { imaging for location) }\end{array}$ & $\begin{array}{l}\text { Involvement of foramen of Luschka, IVth ventricle, cisterna magna, and } \\
\text { CPA was seen in WNT-MB } \\
\text { In } 87 \% \text { of patients, contrast enhancement involved entire tumor } \\
\text { Intra-tumoral hemorrhage was seen in } 31 \% \text { of patients }\end{array}$ \\
\hline Keil et al. ${ }^{[27]}, 2017$ & Adult MB & 28 & $\begin{array}{l}\text { Conventional MRI } \\
\text { features }\end{array}$ & $\begin{array}{l}\text { Absence of hydrocephalus, macrometastases, and hemorrohage was } \\
\text { suggestive of WNT-MB } \\
\text { SHH-MB was associated with larger tumor volume and edema } \\
\text { Contact with lower rhombic lip and hemorrhage were relatively common } \\
\text { in group } 4 \text { tumors }\end{array}$ \\
\hline Zhao et al. ${ }^{[28]}, 2017$ & Adult MB & 125 & $\begin{array}{l}\text { Conventional MRI } \\
\text { features }\end{array}$ & $\begin{array}{l}\text { Large majority of } \mathrm{SHH}-\mathrm{MB} \text { were hemispheric in location } \\
48 \% \text { of } \mathrm{SHH}-\mathrm{MB} \text { had burden exclusively in rostral cerebellum } \\
\text { Peri-tumoral edema was seen commonly in } \mathrm{SHH} \text { and WNT-MB } \\
\text { Group } 4 \text { tumors were predominant midline vermian in location } \\
\text { Minimal or no enhancement was seen in } 50 \% \text { of group } 4 \text { tumors }\end{array}$ \\
\hline $\begin{array}{l}\text { Mata-Mbemba } \\
\text { et al. }{ }^{[29]}, 2018\end{array}$ & Pediaric MB & 119 & $\begin{array}{l}\text { Conventional MRI } \\
\text { features and diffusion } \\
\text { imaging }\end{array}$ & $\begin{array}{l}\text { Primary tumors in } \mathrm{SHH}-\mathrm{MB} \text { were hemispheric in location } \\
\text { Primary tumors were smaller }(<3.5 \mathrm{~cm}) \text { in group } 3 \mathrm{MB} \text { compared to group } \\
4 \text { and } \mathrm{SHH}-\mathrm{MB} \\
\text { Laminar metastates were commonly seen in group } 3 \text { tumors } \\
\text { Nodular metastases were commonly seen in group } 4 \text { tumors } \\
\text { Suprasellar/infundibular metastases were specific for group } 4 \mathrm{MB}\end{array}$ \\
\hline $\begin{array}{l}\text { Zapotocky et al. }{ }^{[30]}, \\
2018\end{array}$ & $\begin{array}{l}\text { Metastatic } \\
\text { MB }\end{array}$ & 40 & $\begin{array}{l}\text { Conventional MRI } \\
\text { features and diffusion } \\
\text { imaging }\end{array}$ & $\begin{array}{l}\text { Cerebellar peripheral location was very common in SHH-MB } \\
\text { Minimal enhancement was seen in primary group } 4 \text { tumor } \\
\text { CPA location was seen more commonly in WNT-MB } \\
\text { Spinal metastases were more commonly seen in group } 3 \text { tumors } \\
\text { Ependymal metastasis with restricted diffusion but no enhancement } \\
\text { ("mismatch pattern") was seen in group } 4 \text { tumors (particualrly if located } \\
\text { in the infundibular recess) }\end{array}$ \\
\hline $\begin{array}{l}\text { Dasgupta et al. }{ }^{[31]}, \\
2018\end{array}$ & $\begin{array}{l}\text { Pediatric and } \\
\text { adult MB }\end{array}$ & 111 & $\begin{array}{l}\text { Conventional } \\
\text { MRI features and } \\
\text { developed MRI- } \\
\text { based nomograms } \\
\text { for prediction of } \\
\text { subgroups }\end{array}$ & $\begin{array}{l}\text { WNT-MB was asoociated with smaller tumor size, homogeneous contrast } \\
\text { uptake, and intratumoral hemorrhage } \\
\text { SHH-MB was more likely to have lateralised and superior location, away } \\
\text { from brainstem and presence of peri-tumoral edema } \\
\text { Group } 3 \text { tumors were in midline location, with "fluffy" enhancement and } \\
\text { higher incidence of metatstatic diasease } \\
\text { Group } 4 \text { tumors were in midline and inferior in location with dilatation of } \\
\text { superior recess of IVth ventricle; showed minimal or "patchy" contrast } \\
\text { enhancement }\end{array}$ \\
\hline
\end{tabular}

MRI: magnetic resonance imaging; MRS: magnetic resonance spectroscopy; MB: medulloblastoma; WNT: wingless; SHH: sonic hedge hog; ADC: apparent diffusion co-efficient; CP: cerebellar peduncle; CPA:cerebellopontine angle 
Table 2. Typical imaging characteristics of individual molecular subgroups of medulloblastoma

\begin{tabular}{|c|c|c|c|c|}
\hline MRI features & WNT-MB & SHH-MB & Group 3 MB & Group 4 MB \\
\hline $\begin{array}{l}\text { Location (horizontal } \\
\text { axis) }\end{array}$ & $\begin{array}{l}\text { Midline, but commonly extends to } \\
\text { CP/CPA cistern }\end{array}$ & $\begin{array}{l}\text { Lateralised location involving } \\
\text { hemispheres; midline location } \\
\text { common in infants/young children }\end{array}$ & $\begin{array}{l}\text { Midline location } \\
\text { involving the IVth } \\
\text { ventricle/vermis }\end{array}$ & $\begin{array}{l}\text { Midline location } \\
\text { involving the IVth } \\
\text { ventricle/vermis }\end{array}$ \\
\hline $\begin{array}{l}\text { Location (vertical } \\
\text { axis) }\end{array}$ & $\begin{array}{l}\text { Central in location, but can extend } \\
\text { inferiorly sometimes }\end{array}$ & $\begin{array}{l}\text { Superior location highly specific } \\
\text { (often reaching or abutting the } \\
\text { tentorium) }\end{array}$ & $\begin{array}{l}\text { Central in location, but } \\
\text { can extend inferiorly also }\end{array}$ & $\begin{array}{l}\text { Inferior in location with } \\
\text { dilatation of superior } \\
\text { recess of IVth ventricle }\end{array}$ \\
\hline $\begin{array}{l}\text { Relation with dorsal } \\
\text { brainstem }\end{array}$ & $\begin{array}{l}\text { Often seen infiltrating the dorsal } \\
\text { brainstem }\end{array}$ & $\begin{array}{l}\text { Over } 50 \% \text { tumors away from } \\
\text { dorsal brainstem }\end{array}$ & $\begin{array}{l}\text { Closely related and abuts } \\
\text { the dorsal brainstem }\end{array}$ & $\begin{array}{l}\text { Closely related and abuts } \\
\text { the dorsal brainstem }\end{array}$ \\
\hline $\begin{array}{l}\text { Contrast- } \\
\text { enhancement }\end{array}$ & $\begin{array}{l}\text { Homogeneous bright enhancement } \\
\text { involving majority of the tumor }\end{array}$ & $\begin{array}{l}\text { Variable pattern with moderate } \\
\text { enhancement }\end{array}$ & $\begin{array}{l}\text { Heterogeneous "fluffy" } \\
\text { type of enhancement }\end{array}$ & $\begin{array}{l}\text { Heterogeneous "patchy" } \\
\text { type of enhancement }\end{array}$ \\
\hline $\begin{array}{l}\text { T2-weighted } \\
\text { characteristics }\end{array}$ & $\begin{array}{l}\text { Mostly isointense and } \\
\text { homogeneous }\end{array}$ & $\begin{array}{l}\text { Mostly isointense and } \\
\text { heterogeneous }\end{array}$ & $\begin{array}{l}\text { Mostly hypointense and } \\
\text { homogeneous }\end{array}$ & $\begin{array}{l}\text { Hyperintense/isointense } \\
\text { and homogeneous }\end{array}$ \\
\hline Peri-tumoral edema & Mild or absent & $\begin{array}{l}\text { Significant edema, often }>1.5 \mathrm{~cm} \\
\text { beyond the tumor }\end{array}$ & Absent or mild & Absent or mild \\
\hline $\begin{array}{l}\text { Intra-tumoral } \\
\text { hemorrhage }\end{array}$ & Can be present & Absent & Absent & Absent \\
\hline $\begin{array}{l}\text { Cyst (size and } \\
\text { location) }\end{array}$ & Microcysts; intra-tumoral & $\begin{array}{l}\text { Microcysts \& macrocysts; intra- } \\
\text { tumoral, peri-tumoral }\end{array}$ & Macrocyst; peri-tumoral & $\begin{array}{l}\text { Microcysts; intra- } \\
\text { tumoral }\end{array}$ \\
\hline Hydrocephalus & $\begin{array}{l}\text { Generally absent (if present, mild } \\
\text { to moderate) }\end{array}$ & $\begin{array}{l}\text { Seldom seem (if present, mild to } \\
\text { moderate) }\end{array}$ & $\begin{array}{l}\text { Moderate to severe } \\
\text { hydrocephalus }\end{array}$ & $\begin{array}{l}\text { Moderate to severe } \\
\text { hydrocephalus }\end{array}$ \\
\hline $\begin{array}{l}\text { Metastases } \\
\text { (incidence, location, } \\
\text { and pattern) }\end{array}$ & Absent (rarely, if ever seen) & $\begin{array}{l}\text { Variable incidence } \\
\text { Spinal \& posterior fossa } \\
\text { Possible multi-centricity }\end{array}$ & $\begin{array}{l}\text { Highest incidence } \\
\text { Spinal metastases } \\
\text { Laminar metastases }\end{array}$ & $\begin{array}{l}\text { Moderate incidence } \\
\text { Suprasellar/infundibular } \\
\text { Nodular metastases }\end{array}$ \\
\hline
\end{tabular}

MRI: magnetic resonance imaging; MB: medulloblastoma; WNT: wingless; SHH: sonic hedge hog; CP/CPA: cerebellar peduncle/ cerebellopontine angle
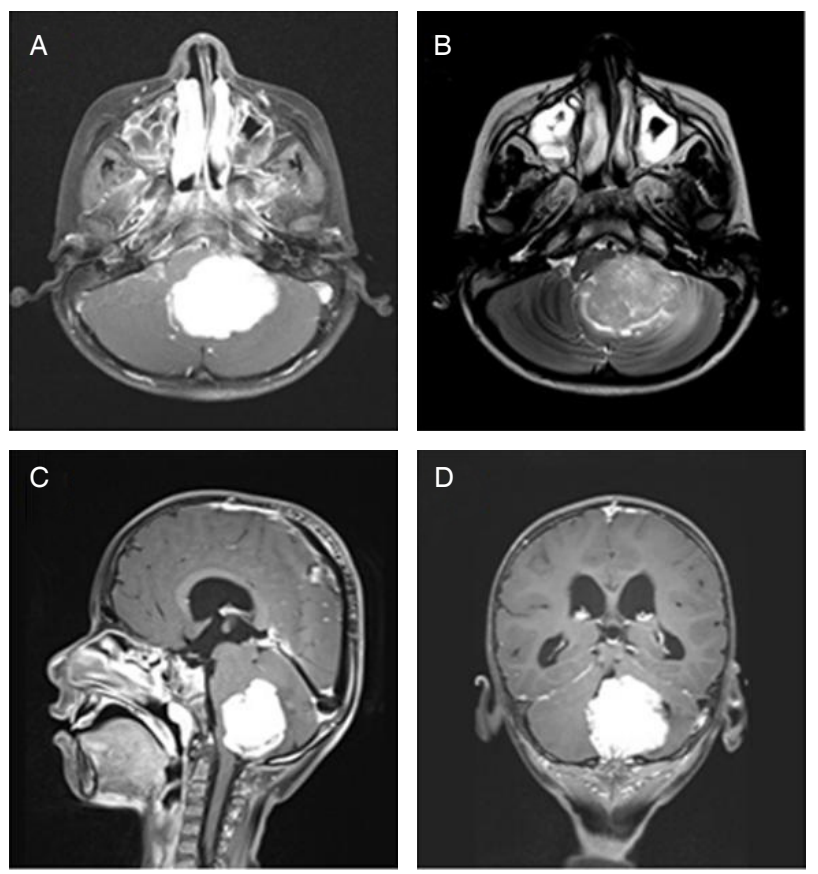

Figure 1. Typical magnetic resonance imaging features of WNT-subgroup medulloblastoma. Post-contrast axial T1-weighted (A), corresponding T2-weighted (B), sagittal (C), and coronal (D) T1-weighted images show a well-defined, lobulated, homogeneous and intensely enhancing tumor arising in the midline vermian region with extension towards the left cerebellopontine angle

the CPA in $20 \%$ of patients. A study involving 17 patients with WNT-pathway medulloblastoma, Dasgupta and colleagues ${ }^{[31]}$ reported the epicentre to be in the midline in the horizontal axis in $77 \%$ of patients; the remaining $23 \%$ showed extension along the cerebellar peduncle towards the foramen of Luschka and/or CPA. They also reported central $(71 \%)$ or inferior $(23 \%)$ location of these tumors on the vertical axis in the vast majority, with only $6 \%$ of patients having a rostral location. 

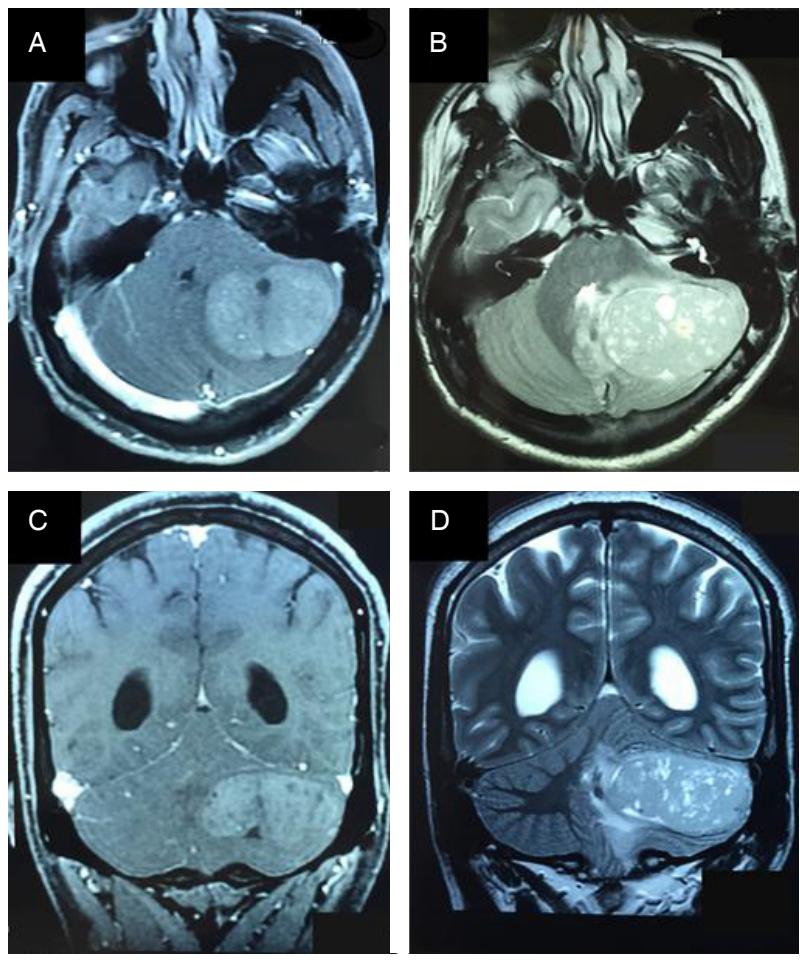

Figure 2. Typical magnetic resonance imaging features of sonic hedge hog-subgroup medulloblastoma. Post-contrast axial T1-weighted (A) and corresponding T2-weighted (B) images show the tumor to be located peripherally in the left cerebellar hemisphere reaching the surface. The tumor extends superiorly to abut the tentorium on post-contrast coronal T1-weighted (C) and corresponding T2-weighted (D) images. Note the presence of intra-tumoral cysts and moderate peri-tumoral edema
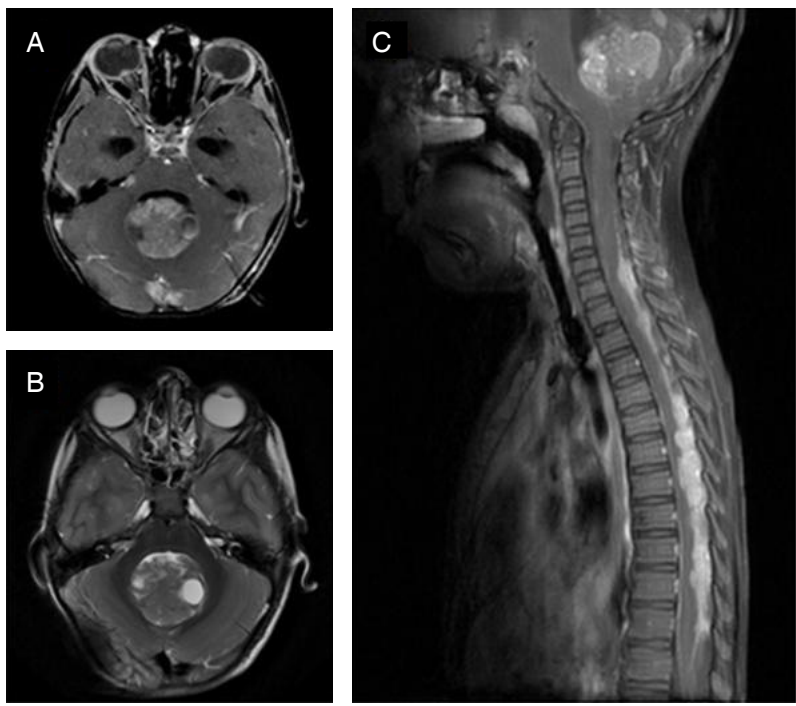

Figure 3. Typical magnetic resonance imaging features of group 3 medulloblastoma. Post-contrast axial T1-weighted (A) and corresponding T2-weighted (B) images show a midline posterior fossa mass filling the IVth ventricle with "cotton-wool" pattern of enhancement. Post-contrast sagittal T1-weighted image (C) shows the inferior location of the primary tumor with widespread leptomeningeal dissemination in the brain and spine

\section{Contrast-enhancement pattern}

WNT-subgroup tumors are usually characterized by intense and homogeneous contrast enhancement throughout the tumor suggesting high vascularity. Patay et al. ${ }^{[26]}$ reported $87 \%$ of WNT-subgroup tumors to have enhancement involving the entire tumor, with only $13 \%$ showing inhomogeneous enhancement. 

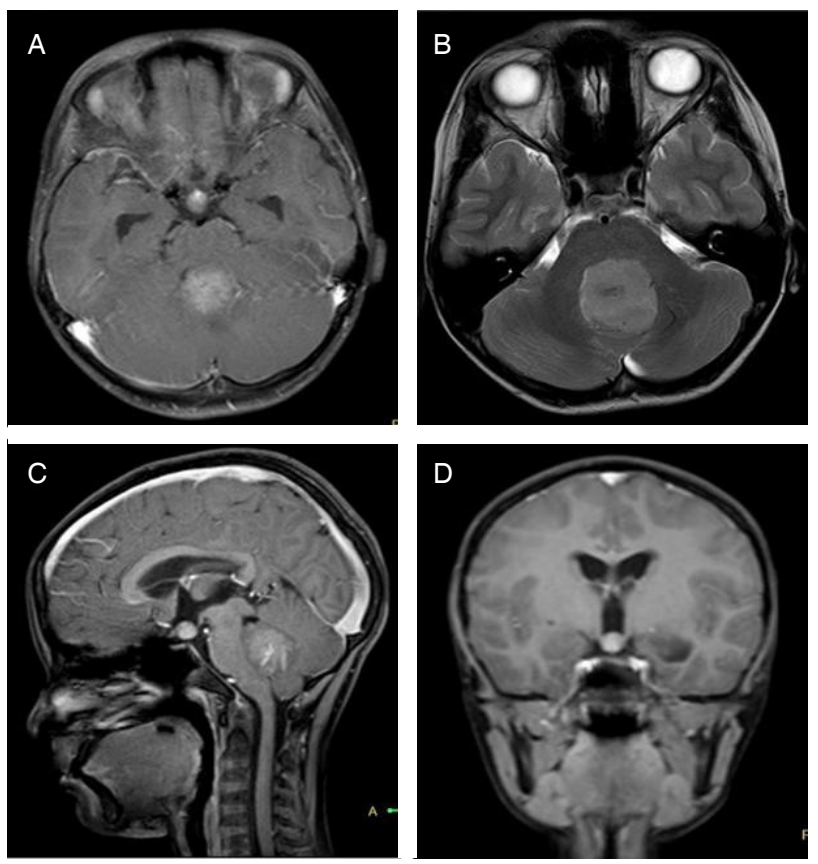

Figure 4. Typical magnetic resonance imaging features of group 4 medulloblastoma. Post-contrast axial T1-weighted (A) image show a mildly enhancing tumor arising in the midline posterior fossa. Corresponding T2-weighted image (B) better delineates the extent of primary tumor. Note the mild dilatation of the superior recess of the IVth ventricle on post-contrast sagittal T1-weighted (C) image and presence of suprasellar/infundibular metastasis best appreciated on the post-contrast coronal T1-weighted (D) image

However, Lastowska et al. ${ }^{[24]}$ reported weak or minimal enhancement in 3 of 6 (50\%) WNT-subgroup medulloblastoma. In their cohort of adult WNT-pathway medulloblastoma, Zhao et al. ${ }^{[28]}$ found significant contrast enhancement in all 17 patients, which was either heterogeneous (82\%) or solid (18\%). In their cohort of 17 patients with WNT-pathway medulloblastoma, Dasgupta et al. ${ }^{[31]}$ reported intense contrast enhancement in all patients; 53\% showed homogeneous enhancement, while the enhancement pattern was inhomogeneous in the remaining $47 \%$ of patients.

\section{Intra-tumoral hemorrhage}

It has been shown from animal studies ${ }^{[34]}$ that paracrine signals from the mutant $\beta$-catenin result in the aberrant fenestrated vasculature in WNT-driven medulloblastoma. The same authors ${ }^{[34]}$ also reported results from two independent cohorts wherein WNT-subgroup tumors were found to be associated with frank hemorrhage in $90 \%$ of patients during surgery as opposed to $12.5 \%$ for the other three subgroups. In the series by Patay et al ${ }^{[26]}$, hemorrhage was appreciated on pre-operative imaging in $31 \%$ of children with WNT-subgroup medulloblastoma $(n=16)$. On the contrary, Keil et al.$^{[27]}$, reported absence of hemorrhage on imaging in all 4 cases of adult WNT-pathway medulloblastoma. However, in another study involving adult patients ${ }^{[28]}$, hemorrhage or mineralization was reported in 11 of 17 (67\%) WNT-pathway medulloblastoma. In the series by Dasgupta et al. ${ }^{[31]}$, hemorrhage was identified on imaging in $18 \%$ patients with WNTsubgroup medulloblastoma $(n=17)$ compared to $<10 \%$ for other subgroups, necessitating the routine use of susceptibility-weighted or gradient-echo imaging for reliable identification.

\section{Other imaging features}

WNT subgroup tumors are usually smaller in size and volume compared to other subgroups which may be reflective of slow growth kinetics and indolent biology. In their series of adult medulloblastoma, Keil et al. ${ }^{[27]}$ reported the median tumor volume to be $5.6 \mathrm{~cm}^{3}$ for WNT-subgroup tumors compared to $30.6 \mathrm{~cm}^{3}$ and $25 \mathrm{~cm}^{3}$ for SHH and group 4 medulloblastoma respectively. The authors also reported the absence of hydrocephalus, macrometastasis, and hemorrhage as having very high specificity and positive predictive 
value for WNT-subgroup medulloblastoma. Dasgupta et al ${ }^{[31]}$ reported the longest tumor dimension to be < $4 \mathrm{~cm}$ in $>35 \%$ of WNT-subgroup tumors compared to $>4 \mathrm{~cm}$ in $>80 \%$ of patients of other subgroups. The presence of cyst or cavity $(71 \%)$ and perilesional edema (53\%) can also be encountered frequently in adult WNT-subgroup medulloblastoma ${ }^{[28]}$.

\section{SHH SUBGROUP MEDULLOBLASTOMA}

This subgroup comprising nearly $30 \%$ of all medulloblastomas is the most heterogeneous molecular subgroup in terms of genetic aberrations, histologic features, clinical presentation, and outcomes ${ }^{[4-6]}$. Normal cerebellar development is highly dependent on SHH signaling; however, unrestricted SHH activity can lead to medulloblastoma tumorigenesis. The 5-year overall survival in patients with SHH-subgroup medulloblastoma ranges from 50\%-85\% depending upon age at diagnosis, histological subtype, presence of metastases, and underlying molecular genetic abnormalities ${ }^{[3,6]}$. A large proportion $(>50 \%)$ of SHH-pathway medulloblastomas show desmoplastic histology ${ }^{[5,6]}$ with extensive nodularity seen almost exclusively in this subgroup; remaining tumors show either classic or LCA histology. They are seen at comparable frequency in males and females, but have a unique bimodal age incidence with most cases involving infants ( $<3$ years) or adults ( $>18$ years).

\section{Anatomic location}

SHH-pathway medulloblastomas are known to arise from granule neuron precursor cells in the external granular layer of the cerebellum ${ }^{[32,33]}$ and often associated with a lateralized hemispheric location. In the series by Teo et al. ${ }^{[21]}, 9$ of 17 (53\%) SHH tumors were located laterally in the cerebellar hemispheres. Because of the lateralized location, these tumors are often seen to be away from the brainstem with distinct space appreciated between the tumor margin and the dorsal brainstem. A similar observation was reported by Wefers et al. ${ }^{[23]}$, with only 10 of 21 (48\%) SHH-pathway medulloblastomas having any contact with the brainstem. They further noted that for hemispheric location, SHH-subgroup tumors were relatively more rostral (superior) compared to more caudal (inferior) location for WNT-subgroup tumors, and no specific predilection in non-WNT/non-SHH tumors. In a large cohort of adult medulloblastomas ${ }^{[28]}$, exclusive cerebellar location was reported in 40 of 64 (63\%) SHH-pathway tumors, with only 11 (17\%) SHH-tumors being limited to midline vermian location. Zhao et al ${ }^{[28]}$ also reported that $48 \%$ of adult $\mathrm{SHH}$-subgroup medulloblastoma burden exclusively in the rostral cerebellum with only $25 \%$ of SHH-subgroup tumors having any brainstem contact, mostly with the cuneate nucleus. On comparing the anatomic location of adult SHH-subgroup tumors with their infantile and childhood counterparts, the proportion of patients with cerebellar hemispheric location increased with age and was seen in $13 \%, 56 \%$, and $63 \%$ of infantile, childhood, and adult SHH-medulloblastoma respectively. Dasgupta and colleagues ${ }^{[31]}$, also reported lateralized hemispheric location in 29 of $44(66 \%)$ patients with SHH-subgroup medulloblastoma including rising proportion of patients with hemispheric tumors with increasing age at initial diagnosis. Thus, lateralized location was seen in $72 \%$ of adults ( $\geq 18$ years) with SHH-pathway medulloblastoma compared to only $27 \%$ hemispheric location in the infantile age group ( $<3$ years), who continue to have predominantly midline SHH-subgroup tumors. Hemispheric location was seen in nearly $60 \%$ of childhood SHH-pathway medulloblastoma. The authors also reported superior location abutting and/or reaching the tentorium as a specific imaging feature of SHH-subgroup medulloblastoma seen in $48 \%$ of patients, as opposed to only $6 \%$ for all other subgroups.

\section{Contrast-enhancement pattern}

The degree and pattern of contrast-enhancement have been reportedly variable in SHH-pathway medulloblastoma. Lastowska et al. ${ }^{[24]}$ reported 7 of 8 SHH-subgroup tumors to be associated with contrast uptake, which was more frequent compared to other subgroups. Although most adult patients (91\%) with SHH-pathway medulloblastoma do show moderate or intense contrast enhancement, nearly $9 \%$ of patients may show minimal or no enhancement at all ${ }^{[28]}$. Dasgupta et al ${ }^{[31]}$ reported some contrast- 
enhancement in $94 \%$ of patients with SHH-subgroup medulloblastoma, with approximately half of them showing enhancement involving $>80 \%$ of the tumor. Over $50 \%$ of SHH-subgroup tumors demonstrated heterogeneous intensity of contrast uptake within the tumor.

\section{Peri-tumoral edema}

SHH-pathway tumors are more frequently associated with peri-tumoral edema, appreciated as hyperintensity on T2/FLAIR images beyond the tumor margin, compared to other molecular subgroups. In a study of adult medulloblastoma, Keil et al. ${ }^{[27]}$ reported the median volume of edema for SHH-subgroup tumors as $5.1 \mathrm{~cm}^{3}$ compared to $1.2 \mathrm{~cm}^{3}$ for group 4 medulloblastoma. Zhao et al. ${ }^{[28]}$ also reported very frequent presence of peri-tumoral edema in adult SHH-subgroup medulloblastoma, which was seen in 52 of 64 (81\%) patients. In another study ${ }^{[31]}$, 91\% of SHH-subgroup medulloblastoma $(n=44)$ were associated with edema as compared to $26 \%-41 \%$ for the other three subgroups. More importantly, moderate to severe peri-tumoral edema (defined as edema beyond $1.5 \mathrm{~cm}$ from the tumor edge) was almost exclusively seen in this subgroup ( $39 \%$ in $\mathrm{SHH} v \boldsymbol{s}$. $6 \%$ in WNT-subgroup vs. none in non-WNT/non-SHH tumors).

\section{Other imaging features}

$\mathrm{SHH}$-subgroup medulloblastomas are often associated with the presence of both microcyst $(\leq 1 \mathrm{~cm})$ as well as macrocysts $(>1 \mathrm{~cm})$. In a study involving adult $\mathrm{SHH}$-subgroup patients ${ }^{[28]}$, intra-tumoral cyst/cavity was seen in 44 of $64(68 \%)$ patients, second only to WNT-pathway medulloblastoma. Although not classical for SHHpathway, intra-tumoral macrocysts were more commonly seen in infantile SHH-subgroup medulloblastoma ${ }^{[31]}$. The spectral pattern of SHH-subgroup medulloblastoma differs markedly from group 3 and group 4 tumors. SHH-subgroup medulloblastoma are characterized by prominent choline and lipid peaks with low creatine levels and near or complete absence of taurine ${ }^{[25]}$. Using a 5-metabolite signature from magnetic resonance spectroscopy (MRS), Bluml et al. ${ }^{[25]}$ could reliably discriminate the SHH-subgroup from non-SHH medulloblastoma. A proportion of patients with SHH-subgroup medulloblastoma present with enhancing nodular deposits within the cerebellar cortex outside the primary site ${ }^{[29,30]}$; these are now believed to represent multi-centric disease rather than metastases and can identify SHH-subgroup with high specificity.

\section{GROUP 3 MEDULLOBLASTOMA}

Group 3 tumors comprising about 25\% of all medulloblastomas are generally aggressive tumors and have the worst prognosis amongst all subgroups with a 5 -year survival rarely exceeding $50 \%^{[3-6]}$. Although most group 3 tumors exhibit classic morphology, LCA histology is overrepresented in this subgroup (nearly $40 \%$ ), whereas desmoplastic histology is almost never seen. They have a male preponderance (twice as common in boys compared to girls), occur mostly in younger children (almost never in adults) and have a high incidence of leptomeningeal metastases (40\%-50\%) at initial diagnosis ${ }^{[5,6]}$.

\section{Anatomic location}

Unlike WNT and SHH-subgroup medulloblastoma, there is limited understanding of the tumorigenesis in group 3 and 4 tumors $^{[33]}$. It is hypothesized that group 3 medulloblastomas arise from prominin $1+/ C D 133+$ lineage neural stem cell following decoupling of proliferation and differentiation ${ }^{[5]}$. Nonetheless, group 3 medulloblastomas have been uniformly reported to be in the midline within the IVth ventricle and vermis. Wefers et al. ${ }^{[23]}$, reported that $73 \%$ of group 3 tumors $(n=15)$ grew within the vermis and $80 \%$ were in contact with cochlear and cuneate nucleus. Dasgupta and colleagues ${ }^{[31]}$, reported that even on the vertical axis, majority $(70 \%)$ of these tumors $(n=27)$ were central in location, with only $30 \%$ having the epicentre somewhat inferiorly.

\section{Contrast-enhancement pattern}

While most of the other conventional imaging features are non-specific for group 3 tumors, contrast enhancement assumes significance in differentiating them from other subgroups. Lastowska et al. ${ }^{[24]}$, 
reported extensive contrast enhancement (defined as enhancement in $>75 \%$ of tumor volume) to be significantly correlated with group 3 tumors. They also reported that non-WNT/non-SHH subgroup tumors showing extensive contrast enhancement were associated with poor survival. Mata-Mbemba et al. ${ }^{[29]}$, reported good contrast enhancement in 22 of 26 (86\%) patients with group 3 medulloblastoma; only 4 (14\%) patients showed minimal enhancement. Dasgupta et al. ${ }^{[31]}$, reported good contrast enhancement in $96 \%$ of patients with group 3 medulloblastoma $(n=27)$; nearly $50 \%$ patients showed contrast uptake in $>80 \%$ of the tumor. They described "fluffy" or "cotton-wool" pattern of enhancement (bright contrast uptake admixed with relatively poor uptake) as a specific marker of group 3 medulloblastoma.

\section{Other imaging features}

Perreault et al. ${ }^{[22]}$ reported $63 \%$ of group 3 tumors $(n=25)$ to be associated with ill-defined tumor margins compared to other subgroups (nearly 10\%). In the study by Dasgupta et al $.^{[3]}, 67 \%$ of group 3 medulloblastomas $(n=27)$ were classified as having an ill-defined infiltrative margin with no tumor showing a lobulated margin. MRS characteristics ${ }^{[2]}$ of group 3 medulloblastoma include high creatinine levels, readily detectable taurine (generally minimal or absent in SHH) and lower lipid levels (prominent peak in SHH). The sizes of primary tumors in group 3 were reportedly smaller compared to other subgroups (generally $<3.5 \mathrm{~cm}$ ), particularly in the presence of metastases suggestive of an aggressive malignancy with metastatic dissemination as an early clonal event ${ }^{[29,30]}$ that does not give time to the primary tumor to grow locally and fill the IVth ventricle. Metastases from group 3 medulloblastoma are more likely to be spinal, typically laminar, often quite large with "sugar-coating" appearance, and have a matching pattern, i.e., presence of enhancement post-contrast as well as restricted diffusion ${ }^{[29,30]}$.

\section{GROUP 4 MEDULLOBLASTOMA}

Group 4 tumors are the most prevalent subgroup accounting for 35\% of all medulloblastomas and have an intermediate prognosis ${ }^{[5,6]}$. The 5 -year overall survival in group 4 tumors can be highly variable, ranging from $\sim 50 \%$ in patients with poor-risk features (subtotal resection, metastases, LCA histology) to $>85 \%$ in patients with favorable prognostic features ${ }^{[3,6]}$. Group 4 tumors are commonly seen in childhood and early adolescence, and much less commonly in extremes of age (infants or adults). There is a striking male preponderance in this subgroup ${ }^{[5,6]}$ even reaching beyond $80 \%$. They are mostly associated with classic histology, although a significant minority (about 25\%) does show LCA morphology. Metastatic disease is reported in $35 \%-40 \%$ of patients at initial diagnosis (second only to group 3 tumors).

\section{Anatomic location}

Although not clearly known, it is hypothesized that deregulation of synaptic pruning in the premature glutamatergic neuronal network resulting in an imbalance between apoptosis, survival and proliferation, is the likely underlying mechanism for tumorigenesis in group 4 medulloblastoma ${ }^{[5]}$. Group 4 tumors are exclusively known to be located in the midline in the IVth ventricle. Wefers et al ${ }^{[23]}$ found 23 of 27 (90\%) group 4 tumors to be located in the midline and having contact with the cochlear and cuneate nucleus. In a study of adult medulloblastoma, Zhao et al. ${ }^{[28]}$ reported predominant midline vermian location of group 4 tumors in 39 of 44 $(89 \%)$ patients and brainstem contact with caudate and cuneate nucleus in $36(82 \%)$ patients. In the study by Dasgupta and colleagues ${ }^{[31]}$, all group 4 tumors $(n=23)$ were located in the midline on the horizontal axis. Nearly $39 \%$ of these tumors had their epicentre located inferiorly on the vertical axis (some even extending beyond the foramen magnum), which was highest amongst all subgroups. The authors also reported a very specific imaging feature of group 4 medulloblastoma, i.e., inferior extension with resultant dilation of the superior recess of IVth ventricle.

\section{Contrast-enhancement pattern}

Weak, minimal, or no enhancement is a characteristic feature of group 4 tumors, which serves as an important feature to distinguish from other subgroups. Perreault et al. ${ }^{[22]}$ reported $59 \%$ of group 4 
medulloblastoma ( $n=36$ ) as having no or minimal enhancement as opposed to $7 \%$ for other subgroups. Similarly, Lastowska et al ${ }^{[24]}$ reported 6 of $10(60 \%)$ group 4 tumors with weak or no enhancement. In a large study of adult medulloblastoma, Zhao et al. ${ }^{[28]}$, reported minimal or no enhancement in 22 of 44 (50\%) patients with group 4 tumors. Mata-Mbemba et al. ${ }^{[29]}$ reported minimal enhancement of the primary tumor in $55 \%$ of patients with group 4 medulloblastoma $(n=49)$. Dasgupta et al. ${ }^{[31]}$ reported minimal or no enhancement in $30 \%$ of group 4 medulloblastoma $(n=23)$. Even for tumors showing partial contrast uptake, intervening non-enhancing areas gave rise to a "patchy" enhancement pattern.

\section{Other imaging features}

The MRS profile ${ }^{[25]}$ of group 4 tumors is quite similar to group 3, but significantly different from SHHsubgroup medulloblastoma, as described earlier. Similar to group 3 medulloblastoma, the majority (70\%) of group 4 tumors do not show any peri-tumoral edema ${ }^{[3]}$. The location, morphology, and imaging characteristics of metastases from group 4 medulloblastoma are quite distinct ${ }^{[29,30]}$. Metastatic deposits from group 4 tumors are more nodular and associated with a "mismatching pattern", i.e., lack of or minimal contrast enhancement but restricted diffusion. The presence of metastases in the suprasellar region and/or infundibular recess of the third ventricle is a highly specific marker of group 4 medulloblastoma ${ }^{[29,30]}$.

\section{PREDICTORS OF MOLECULAR SUBGROUPS}

The presence of subgroup-specific imaging features has prompted researchers to develop models and nomograms for accurate pre-operative prediction of molecular subgroups in medulloblastoma. In a large cohort involving 125 patients $^{[28]}$, researchers from Beijing Tian Tan Hospital in China reported location (midline vermis/IVth ventricle, cerebellar hemisphere, and CPA; $P<0.0001$ ) and pattern of enhancement; $P=0.0048$ ) to be independent predictors of molecular subgrouping in adult medulloblastoma. With the logistic regression model based on location and pattern of enhancement, $79 \%$ of adult medulloblastomas were accurately and appropriately classified, including WNT (24\%), SHH (86\%), and group 4 (91\%) medulloblastoma (R-squared goodness of fit $=0.669$ ). The accuracy of the logistic regression model improved significantly after incorporating anatomic localization patterns (horizontal location, brainstem contact and vertical location) with pattern of enhancement. With the modified model, $92 \%$ of adult medulloblastomas could be accurately and appropriately classified, including WNT (65\%), SHH (95\%), and group 4 (98\%) medulloblastoma $(\mathrm{R}$-squared goodness of fit $=0.795)$.

Using a step-wise, multi-variable, multi-nomial, logistic regression model in a large single-institution cohort $(n=119)$, researchers from Hospital for Sick Children at the University of Toronto ${ }^{[29]}$, demonstrated the lateralized cerebellar location for SHH-subgroup with an adjusted odds ratio (aOR) of $9(P<0.0001)$; minimal enhancement of primary tumor for group 4 medulloblastoma $(\mathrm{aOR}=5.2, P<0.0001)$; CPA location for WNTpathway medulloblastoma $(\mathrm{aOR}=1.4, P<0.03)$; ependymal metastases with mismatching pattern for group 4 tumor $(\mathrm{aOR}=2.8, P<0.001)$; and spinal leptomeningeal metastases for group 3 tumors $(\mathrm{aOR}=1.9, P<0.01)$ were independent predictors of molecular subgrouping. Specifically, the presence of a metastasis in the third ventricular infundibular recess showing mismatching pattern was significantly associated with group 4 disease $(P<0.02)$.

In parallel, researchers from Tata Memorial Centre in India ${ }^{[31]}$ recently reported on the radiogenomics of medulloblastoma in their single-institution cohort of 111 patients with known molecular subgroup affiliation. Amongst their panel of 19 pre-specified imaging features, 11 were differentially distributed across the four subgroups with statistical significance $(P<0.05)$ on univariate analysis. Two-thirds of patients $(n=76)$ were chosen randomly from individual subgroups to form the training cohort (TC), while the remaining one-third $(n=35)$ constituted the validation cohort (VC). Multi-nomial logistic regression analysis was performed in the TC to identify few imaging features with highest discrimination of one subgroup from the other three subgroups to construct subgroup-specific binary nomograms. Using receiver operating 
characteristics analysis, the area under the curve (AUC) was also generated to assess the reliability of the nomograms. The predictive accuracy of SHH-subgroup nomogram (location on horizontal and vertical axis, relationship with dorsal brainstem, heterogeneity of contrast-enhancement, and peri-tumoral edema as the discriminating imaging features) was the highest followed by group 4-specific nomogram. Group 3 and WNT-subgroup nomograms had suboptimal predictive accuracy. For a sensitivity and specificity $>85 \%$ each, a cut-off total score of 13.3 was obtained from the SHH-specific nomogram in the TC. When this was tested in the VC, $93 \%$ of SHH-subgroup tumors still had total score above this threshold (13.3) as opposed to only $5 \%$ of non-SHH tumors for a sensitivity of $93 \%$ and specificity of $95 \%$. The nomogram identified even midline SHH tumors with acceptable accuracy. AUC for the SHH subgroup was excellent, with a value of 0.939 and 0.991 in TC and VC respectively.

\section{PRACTICAL AND CLINICAL IMPLICATIONS}

With identification of different molecular subgroups, medulloblastoma is no longer considered a single entity $^{[4-6]}$, but represents a heterogeneous group of diseases with a widely variable spectrum, mandating optimization of therapy within individual subgroups. In the past two decades, post-operative adjuvant treatment in medulloblastoma has been largely based on a clinico-radiological risk-stratification system ${ }^{[35]}$ wherein patients over the age of 3 years at initial diagnosis with post-operative residual tumor $<1.5 \mathrm{~cm}^{2}$ (Ro) and absence of metastases (Mo) were classified as having average-risk/standard-risk disease. High-risk disease was defined as the presence of any one of the following features viz. age $<3$ years, residual tumor $\geq 1.5 \mathrm{~cm}^{2}(\mathrm{R}+)$, and any evidence of metastases $\left(\mathrm{M}_{+}\right)$. This traditional clinico-radiological risk-classification has now been supplanted by a more contemporary consensus risk-stratification schema (in the molecular era) into low-risk, standardrisk, high-risk, and very high-risk categories with expected long-term overall survival > 90\%, > 75\%-90\%, > $50 \%-75 \%$, and $<50 \%$ respectively ${ }^{[36]}$. Long-term treatment-related morbidity in medulloblastoma including neuro-cognitive dysfunction is largely dependent upon the dose and volume of irradiation ${ }^{[37,38]}$.

Cerebellar mutism, motor deficits, and intellectual impairment can be differentially prevalent in individual molecular subgroups ${ }^{[39]}$, likely based on anatomic location of tumor and intensity of therapy. Consequently, there is significant potential of de-intensification of treatment in children with low-risk disease (WNTpathway), while further intensification of treatment may be more appropriate for patients in the high-risk/ very high-risk category (metastatic group 3 or 4 tumors). The identification of specific genetic alterations (PTCH1 or SUFU mutations) may allow the use of targeted therapies in particular subgroups (SHH-pathway), leading to a more personalized approach in the future ${ }^{[5,6]}$.

Tissue-based information remains the gold-standard for histo-morphology and molecular subgrouping in medulloblastoma that cannot be replaced by any imaging-based classification. However, this tissuebased information is not available pre-operatively to the operating neuro-surgeon, but only after surgical resection of the tumor. The prognostic benefit of increased extent of resection becomes highly attenuated after taking molecular subgroup affiliation into account. In a retrospective multi-institutional cohort involving 787 patients $^{[40]}$, there was no significant survival benefit of greater extent of resection (gross total/ near total versus sub-total resection) for WNT, SHH, and group 3 tumors. Only in group 4 tumors, gross total/near total resection was associated with significant benefit in progression-free survival compared to sub-total resection, but not in overall survival. The authors concluded that although maximal safe resection should remain the standard of care, aggressive neuro-surgical removal of small residual portions of medulloblastoma should not be attempted, particularly when the anticipated morbidity is high. Several different studies discussed herein, some of which were summarized in a review previously suggest that MRI features can be a helpful and promising tool for early identification of molecular subgrouping in medulloblastoma with potential to influence therapy ${ }^{[41]}$. The availability of robust and reliable imagingbased predictors of molecular subgrouping could aid neuro-surgical decision-making. The clinical utility of tissue-based subgrouping, though undeniable, is greatly dependent upon the availability of resources (expertise and infrastructure), costs, and turn-around time. The addition of molecular genetic information 
in the 2016 update of the WHO classification ${ }^{[7]}$ for an integrated diagnosis of medulloblastoma should ensure widespread adoption of a practical IHC-based classification in routine clinical practice. While this IHCbased classification ${ }^{[13]}$ identifies WNT and SHH subgroups with high accuracy, it cannot reliably discriminate between group 3 and 4 tumors which are classified collectively as non-WNT/non-SHH medulloblastoma, with differing prognosis. Imaging characteristics (contrast-enhancement of primary tumor and location, morphology, and pattern of metastatic disease) can further help subclassify non-WNT/non-SHH group into group 3 and group 4 tumors with high accuracy ${ }^{[29,30]}$.

Novel insights in the presence of significant heterogeneity within individual molecular subgroups has prompted further subclassification of medulloblastoma ${ }^{[42,43]}$ which is going to be very difficult to be matched even with advanced contemporary imaging methods. Apart from identifying molecular subgroups, imaging biomarkers may serve as putative predictive and prognostic factors in medulloblastoma. The presence of homogenous contrast uptake (WNT-subgroup) may imply uniform disruption of blood-brain barrier leading to better CNS penetration of systemic agents and resultant responsiveness to chemotherapy. Other radiological features have already been reported to be associated with outcomes, e.g., bright enhancement in non-WNT/non-SHH tumors is associated with poor overall survival, while superiorly located SHH-subgroup tumors are associated with higher risk of local recurrence. The advent of deep machine-learning (supervised/ unsupervised) techniques and convoluted artificial neural networks should provide unique opportunities to further improve the accuracy of such radiogenomic correlation and prediction ${ }^{[4,45]}$.

\section{CONCLUSION}

Medulloblastoma is a heterogeneous disease comprising four molecular subgroups with distinct developmental origins, unique transcriptional profiles, diverse phenotypes, and varying clinical outcomes. It has been increasingly recognized that imaging is useful not only for diagnosis and staging, but also may be a reflection of underlying disease biology. The systematic assessment and correlation of imaging features with molecular subgrouping in medulloblastoma are increasingly being reported with potential to serve as independent predictive and prognostic biomarkers in contemporary neuro-oncologic practice.

\section{DECLARATIONS}

\section{Authors' contributions}

Literature search and manuscript writing: Dasgupta A, Gupta T

\section{Availability of data and materials}

Not applicable.

\section{Financial support and sponsorship}

None.

\section{Conflicts of interest}

All authors declared that there are no conflicts of interest.

\section{Ethical approval and consent to participate}

Not applicable.

\section{Consent for publication}

Not applicable.

\section{Copyright}

(c) The Author(s) 2018. 


\section{REFERENCES}

1. Leece R, Xu J, Ostrom QT, Chen Y, Kruchko C, et al. Global incidence of malignant brain and other central nervous system tumors by histology, 2003-2007. Neuro Oncol 2017;19:1553-64.

2. Khanna V, Achey RL, Ostrom QT, Block-Beach H, Kruchko C, et al. Incidence and survival trends for medulloblastomas in the United States from 2001 to 2013. J Neurooncol 2017;135:433-41.

3. Kool M, Korshunov A, Remke M, Jones DT, Schlanstein M, et al. Molecular subgroups of medulloblastoma: an international metaanalysis of transcriptome, genetic aberrations, and clinical data of WNT, SHH, group 3, and group 4 medulloblastomas. Acta Neuropathol 2012;123:473-84.

4. Gajjar A, Bowers DC, Karajannis MA, Leary S, Witt H, et al. Pediatric brain tumors: innovative genomic information is transforming the diagnostic and clinical landscape. J Clin Oncol 2015;33:2986-98.

5. Gupta T, Shirsat N, Jalali R. Molecular subgrouping of medulloblastoma: impact upon research and clinical practice. Curr Pediatr Rev 2015;11:106-19.

6. Taylor MD, Northcott PA, Korshunov A, Remke M, Cho YJ, et al. Molecular subgroups of medulloblastoma: the current consensus. Acta Neuropathol 2012;123:465-72.

7. Louis DN, Perry A, Reifenberger G, von Deimling A, Figarella-Branger D, et al. The 2016 World Health Organization classification of tumors of the central nervous system: a summary. Acta Neuropathol 2016;131:803-20.

8. Northcott PA, Korshunov A, Witt H, Hielscher T, Eberhart CG, et al. Medulloblastoma comprises four distinct molecular variants. J Clin Oncol 2011;29:1408-14.

9. Kool M, Koster J, Bunt J, Hasselt NE, Lakeman A, et al. Integrated genomics identifies five medulloblastoma subtypes with distinct genetic profiles, pathway signatures and clinicopathological features. PLoS One 2008;3:e3088.

10. Cho YJ, Tsherniak A, Tamayo P, Santagata S, Ligon A, et al. Integrative genomic analysis of medulloblastoma identifies a molecular subgroup that drives poor clinical outcome. J Clin Oncol 2011;29:1424-30.

11. Kunder R, Jalali R, Sridhar E, Moiyadi A, Goel N, et al. Real-time PCR assay based on the differential expression of microRNAs and protein-coding genes for molecular classification of formalin-fixed paraffin embedded medulloblastomas. Neuro Oncol 2013;15:1644-51.

12. Ellison DW, Kocak M, Dalton J, Megahed H, Lusher ME, et al. Definition of disease-risk stratification groups in childhood medulloblastoma using combined clinical, pathologic, and molecular variables. J Clin Oncol 2011;29:1400-7.

13. Kaur K, Kakkar A, Kumar A, Mallick S, Julka PK, et al. Integrating molecular subclassification of medulloblastomas into routine clinical practice: a simplified approach. Brain Pathol 2016;26:334-43.

14. Schwalbe EC, Williamson D, Lindsey JC, Hamilton D, Ryan SL, et al. DNA methylation profiling of medulloblastoma allows robust subclassification and improved outcome prediction using formalin-fixed biopsies. Acta Neuropathol 2013;125:359-71.

15. Hovestadt V, Remke M, Kool M, Pietsch T, Northcott PA, et al. Robust molecular subgrouping and copy-number profiling of medulloblastoma from small amounts of archival tumour material using high-density DNA methylation arrays. Acta Neuropathol 2013;125:913-6

16. Koeller KK, Rushing EJ. From the archives of the AFIP: medulloblastoma: a comprehensive review with radiologic-pathologic correlation. Radiographics 2003;23:1613-37.

17. Gupta T, Sarkar C, Rajshekhar V, Chatterjee S, Shirsat N, et al. Indian society of neuro-oncology consensus guidelines for the contemporary management of medulloblastoma. Neurol India 2017;65:315-32.

18. Kuo MD, Jamshidi N. Behind the numbers: decoding molecular phenotypes with radiogenomics--guiding principles and technical considerations. Radiology 2014;270:320-5.

19. Gillies RJ, Kinahan PE, Hricak H. Radiomics: images are more than pictures, they are data. Radiology 2016;278:563-77.

20. Kickingereder P, Andronesi OC. Radiomics, metabolic, and molecular MRI for brain tumors. Semin Neurol 2018;38:32-40.

21. Teo WY, Shen J, Su JM, Yu A, Wang J, et al. Implications of tumor location on subtypes of medulloblastoma. Pediatr Blood Cancer 2013;60:1408-10.

22. Perreault S, Ramaswamy V, Achrol AS, Chao K, Liu TT, et al. MRI surrogates for molecular subgroups of medulloblastoma. AJNR Am J Neuroradiol 2014;35:1263-9.

23. Wefers AK, Warmuth-Metz M, Poschl J, von Bueren AO, Monoranu CM, et al. Subgroup-specific localization of human medulloblastoma based on pre-operative MRI. Acta Neuropathol 2014;127:931-3.

24. Lastowska M, Jurkiewicz E, Trubicka J, Daszkiewicz P, Drogosiewicz M, et al. Contrast enhancement pattern predicts poor survival for patients with non-WNT/SHH medulloblastoma tumours. J Neurooncol 2015;123:65-73.

25. Bluml S, Margol AS, Sposto R, Kennedy RJ, Robison NJ, et al. Molecular subgroups of medulloblastoma identification using noninvasive magnetic resonance spectroscopy. Neuro Oncol 2016;18:126-31.

26. Patay Z, DeSain LA, Hwang SN, Coan A, Li Y, et al. MR imaging characteristics of wingless-type-subgroup pediatric medulloblastoma. AJNR Am J Neuroradiol 2015;36:2386-93.

27. Keil VC, Warmuth-Metz M, Reh C, Enkirch SJ, Reinert C, et al. Imaging biomarkers for adult medulloblastomas: genetic entities may be identified by their MR imaging radiophenotype. AJNR Am J Neuroradiol 2017;38:1892-8.

28. Zhao F, Li C, Zhou Q, Qu P, Wang B, et al. Distinctive localization and MRI features correlate of molecular subgroups in adult medulloblastoma. J Neurooncol 2017;135:353-60.

29. Mata-Mbemba D, Zapotocky M, Laughlin S, Taylor MD, Ramaswamy V, et al. MRI characteristics of primary tumors and metastatic lesions in molecular subgroups of pediatric medulloblastoma: a single-center study. AJNR Am J Neuroradiol 2018;39:949-55.

30. Zapotocky M, Mata-Mbemba D, Sumerauer D, Liby P, Lassaletta A, et al. Differential patterns of metastatic dissemination across 
medulloblastoma subgroups. J Neurosurg Pediatr 2018;21:145-52.

31. Dasgupta A, Gupta T, Pungavkar S, Shirsat N, Epari S, et al. Nomograms based on pre-operative multi-parametric magnetic resonance imaging for prediction of molecular subgrouping in medulloblastoma: results from a radiogenomics study of 111 patients. Neuro Oncol 2018; doi: 10.1093/neuonc/noy093.

32. Gibson P, Tong Y, Robinson G, Thompson MC, Currle DS, et al. Subtypes of medulloblastoma have distinct developmental origins. Nature 2010;468:1095-9.

33. Gilbertson RJ, Ellison DW. The origins of medulloblastoma subtypes. Annu Rev Pathol 2008;3:341-65.

34. Phoenix TN, Patmore DM, Boop S, Boulos N, Jacus MO, et al. Medulloblastoma genotype dictates blood brain barrier phenotype. Cancer Cell 2016;29:508-22.

35. Zeltzer PM, Boyett JM, Finlay JL, Albright AL, Rorke LB, et al. Metastasis stage, adjuvant treatment, and residual tumor are prognostic factors for medulloblastoma in children: conclusions from the Children's Cancer Group 921 randomized phase III study. J Clin Oncol 1999;17:832-45.

36. Ramaswamy V, Remke M, Bouffet E, Bailey S, Clifford SC, et al. Risk stratification of childhood medulloblastoma in the molecular era: the current consensus. Acta Neuropathol 2016;131:821-31.

37. Mulhern RK, Merchant TE, Gajjar A, Reddick WE, Kun LE. Late neurocognitive sequelae in survivors of brain tumours in childhood. Lancet Oncol 2004;5:399-408.

38. Fossati P, Ricardi U, Orecchia R. Pediatric medulloblastoma: toxicity of current treatment and potential role of protontherapy. Cancer Treat Rev 2009;35:79-96.

39. Moxon-Emre I, Taylor MD, Bouffet E, Hardy K, Campen CJ, et al. Intellectual outcome in molecular subgroups of medulloblastoma. J Clin Oncol 2016;34:4161-70.

40. Thompson EM, Hielscher T, Bouffet E, Remke M, Luu B, et al. Prognostic value of medulloblastoma extent of resection after accounting for molecular subgroup: a retrospective integrated clinical and molecular analysis. Lancet Oncol 2016;17:484-95.

41. Colafati GS, Voicu IP, Carducci C, Miele E, Carai A, et al. MRI features as a helpful tool to predict the molecular subgroups of medulloblastoma: state of the art. Ther Adv Neurol Disord 2018;11:1756286418775375.

42. Cavalli FMG, Remke M, Rampasek L, Peacock J, Shih DJH, et al. Intertumoral heterogeneity within medulloblastoma subgroups. Cancer Cell 2017;31:737-54.

43. Schwalbe EC, Lindsey JC, Nakjang S, Crosier S, Smith AJ, et al. Novel molecular subgroups for clinical classification and outcome prediction in childhood medulloblastoma: a cohort study. Lancet Oncol 2017;18:958-71.

44. Parmar C, Grossmann P, Bussink J, Lambin P, Aerts HJ. Machine learning methods for quantitative radiomic biomarkers. Sci Rep 2015;5:13087.

45. Zhou M, Scott J, Chaudhury B, Hall L, Goldgof D, et al. Radiomics in brain tumor: image assessment, quantitative feature descriptors, and machine-learning approaches. AJNR Am J Neuroradiol 2018;39:208-16. 